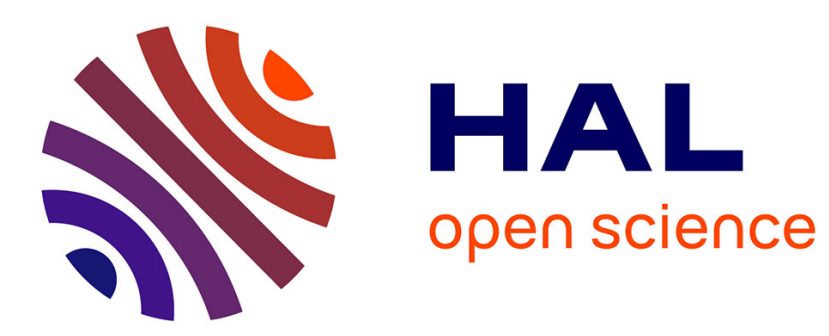

\title{
Modal truncation for linear Hamiltonian systems : a physical energy approach
}

Benjamin Mourllion, Abderazik Birouche

\section{To cite this version:}

Benjamin Mourllion, Abderazik Birouche. Modal truncation for linear Hamiltonian systems: a physical energy approach. Dynamical Systems, 2013, 28 (Issue 2), pp.187-202 (Article). 10.1080/14689367.2013.777397 . hal-00982544

\section{HAL Id: hal-00982544 https://hal.science/hal-00982544}

Submitted on 24 Apr 2014

HAL is a multi-disciplinary open access archive for the deposit and dissemination of scientific research documents, whether they are published or not. The documents may come from teaching and research institutions in France or abroad, or from public or private research centers.
L'archive ouverte pluridisciplinaire HAL, est destinée au dépôt et à la diffusion de documents scientifiques de niveau recherche, publiés ou non, émanant des établissements d'enseignement et de recherche français ou étrangers, des laboratoires publics ou privés. 
Dynamical Systems

Vol. 00, No. 00, September 2012, 1-21

\title{
RESEARCH ARTICLE
}

\section{Modal Truncation for Linear Hamiltonian Systems: a Physical Energy Approach}

\author{
Benjamin Mourllion* and Abderazik Birouche \\ ${ }^{\mathrm{a}} M I P S$ EA 2332 \\ Université de Haute-Alsace - Bât. ENSISA-Lumière \\ 12, rue des frères Lumière. 68093 Mulhouse cedex, France. \\ (Received 00 Month 200x; final version received 00 Month 200x)
}

This paper proposes a new criterion to select the eigenmodes to be left out in a modal truncation procedure. This criterion takes account of the dynamics of the system on the one hand and, on the other hand, the input/output matrices of the system. Therefore, this approach is satisfactory from a system and control theory point-of-view. Moreover, this criterion is physically meaningful because its computation is directly linked with the physical energy supplied to each eigenmode.

Keywords: Modal Analysis; Model Order Reduction; Hamiltonian system; $\mathcal{H}_{\infty}$-norm.

MCS/CCS/AMS Classification/CR Category numbers: 70S05, $78 \mathrm{M} 34$

\section{Notations}

- $M=M^{t} \succ 0 \quad$ (resp. $\left.\succeq 0\right)$ means that $M$ is a symmetric positive-definite matrix (resp. positive-semidefinite).

${ }^{*}$ Corresponding author. Email: benjamin.mourllion@uha.fr

ISSN: 1745-9737 print/ISSN 1745-9745 online

(C) 2012 Taylor \& Francis

DOI: $10.1080 / 1745973 Y Y x x x x x x x x$

http://www.informaworld.com 
- $\operatorname{Re} z, z^{*}$, and $|z|$ denote respectively the real part, the conjugate and the modulus of the complex number $z$.

- $\mathcal{P} f(x)$ denotes the pseudo-function of $f(x)$. When integrating it, one implicitly takes account of the Cauchy principal value (P.V.) of the integral of the considered function. For instance, let $c \in I=[a, b]$ be a singularity of function $f(x)$. The integral over $I$ is undefined whereas the integral of $\mathcal{P} f(x)$ is defined as follows: $\int_{I} \mathcal{P} f(\tau) \mathrm{d} \tau=$ P.V. $\int_{I} f(\tau) \mathrm{d} \tau \triangleq \lim _{\varepsilon \rightarrow 0^{+}}\left(\int_{a}^{c-\varepsilon} f(\tau) \mathrm{d} \tau+\int_{c+\varepsilon}^{b} f(\tau) \mathrm{d} \tau\right)$.

\section{Introduction}

Realistic systems are often complex and characterized by models of a very high order. These models are usually difficult to study in a context of analysis. The interest of model order-reduction is that high order models generate high order controllers/observers leading to numerous complexities and difficulties in synthesis, simulation and implementation. Therefore, it is necessary to reduce the order of these systems. The problem of model order-reduction has become the focus of different research areas (mathematics, mechanics, control theory and computer engineering). Several studies have been devoted to this issue following the class of system considered.

In the control theory domain, state-of-the-art methods for reducing the order of linear models can be found in $[1,2,18]$. One important contribution to model reduction is the balanced truncation method $[8,15]$. In this approach, each state is equally controllable and observable and the reduced order model is obtained by truncating the least controllable and observable states. Balanced truncation is a common method to reduce the order of Linear Time Invariant (LTI) systems thanks to its simplicity of implementation; the stability of the reduced system is guaranteed and the approximation error is bounded. Another important solution to model reduction problems is the optimization approach which minimizes the $\mathcal{H}_{\infty}$-norm (or $\mathcal{H}_{2}$-norm) between the original full order model and the reduced order model $[12,28]$. The main drawback of these solutions is that the reduced models obtained do not necessarily have obvious physical meanings. To overcome this problem, some methods propose several adaptations of the balanced truncation in order to preserve the second-order form of the systems [5, 14, 19]. All these methods cannot be applied to conservative systems where $\mathcal{H}_{\infty}$-norm is infinite. Indeed, in this case, the reachability Gramian and observability Gramian cannot be computed (since the Hankel singular values are infinite), and so, the truncation is not possible. It can be noticed that van der Schaft [23] proposed an alternative approach by associating a gradient (or reciprocal) system with the conservative system [22, 25] but this ad hoc method is not straightforwardly interpretable.

In the mechanical and physical domain, several other methods have been developed, such as Guyan Condensation [10], Component Mode Synthesis [6], Modal Truncation, for example. State-of-the-art and comparative studies of these methods can be found in [11]. 
It is well known that modal truncation helps to keep stronger physical meanings of states but the selection of eigenmodes to be removed is not easy to perform and remains an open issue. As underlined by Wortelboer [27, p. 60], all sorts of criteria for selection have been proposed in literature. Besides, selection criteria are often not made explicit. It is difficult to trace the origins of most modal reduction methods.

This problem will be the topic of the present paper.

The main contribution of this paper is the definition of a new criterion to select the eigenmodes to be left out in the modal truncation step. This criterion is based of the physical energy supplied by the control command and the passivity [24, 25] of physical systems. In order to preserve as much as possible the connection between this new criterion and the physical energy, the Hamiltonian formulation of physical systems will be used.

This paper is organized as follows. Section 2 describes the problems and drawbacks of conventional modal truncation. Section 3, considering classic (i.e. undamped [3, 9, 13]) Hamiltonian systems, presents a strong relation between the energy supplied and the Fourier transform of the input is introduced. This relation leads to the formulation of a new criterion. Two examples of modal energy computation are then presented. The first one deals with a finite-time input signal, while the second one deals with a infinite-time input signal. Section 4 shows how this criterion can be used in the eigenmode selection in modal truncation. One example is studied, with two different modal truncation procedures. Finally, the conclusion will be followed by a few perspectives.

\section{Motivation and Problem Positioning}

\subsection{Introduction}

Generally, modal analysis deals with physical second-order form systems given by:

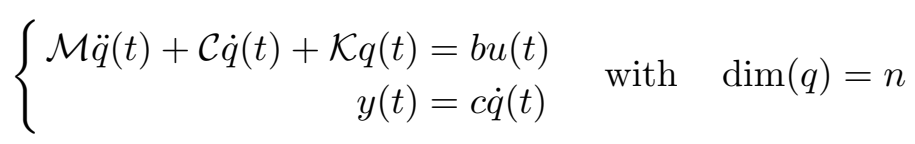

where $\mathcal{M}, \mathcal{C}$ and $\mathcal{K}$ are respectively the mass (or inertia) matrix, damping matrix and stiffness matrix. To ensure the physical feasibility of the second order form system equation (1), these three $n \times n$ matrices have to verify the so-called structural conditions:

- $\mathcal{M}=\mathcal{M}^{t} \succ 0$

- $\mathcal{C}=\mathcal{C}^{t} \succeq 0$

- $\mathcal{K}=\mathcal{K}^{t} \succ 0$ 


\subsection{Modal basis}

As $\mathcal{M}=\mathcal{M}^{t} \succ 0 \Rightarrow \operatorname{det}(\mathcal{M}) \neq 0$, the inertia matrix is invertible and system (1) has $n$ eigenmodes, solution of:

$$
\left(\mathcal{M}^{-1} \mathcal{K}\right) \Phi=\Phi \Omega^{2}
$$

with:

$$
\begin{aligned}
& \Omega=\operatorname{diag}\left\{\omega_{1}, \ldots, \omega_{n}\right\} \text { the eigenfrequencies matrix } \\
& \Phi=\left[\phi_{1}, \ldots, \phi_{n}\right] \text { the modal matrix. }
\end{aligned}
$$

The mass and stiffness matrices are symmetric and positive definite, then the modal matrix is diagonal and the eigenfrequencies are real and positive and the eigenmodes are real. Moreover, the eigenmodes are orthogonal

$$
\phi_{i}^{t} \mathcal{M} \phi_{j}=0 \quad \text { and } \quad \phi_{i}^{t} \mathcal{K} \phi_{j}=0 \quad \text { if } \quad \omega_{i} \neq \omega_{j}
$$

Therefore, the modal transformation gives the following diagonal matrices

$$
\begin{aligned}
\tilde{\mathcal{M}} & =\Phi^{t} \mathcal{M} \Phi \\
\tilde{\mathcal{K}} & =\Phi^{t} \mathcal{K} \Phi
\end{aligned}
$$

Due to the damping matrix, orthogonal modes can be coupled through the following quantity:

$$
\tilde{\mathcal{C}}=\Phi^{t} \mathcal{C} \Phi
$$

If the damping matrix can be written as $\mathcal{C}=a_{m} \mathcal{M}+a_{k} \mathcal{K}$ (known as Rayleigh damping condition [17]) or as $\mathcal{C}=\mathcal{M} \sum_{j=-\infty}^{\infty} a_{j}\left(\mathcal{M}^{-1} \mathcal{K}\right)^{j}$ (known as Caughey damping condition [4]), the modal damping matrix can be written as

$$
\tilde{\mathcal{C}}=\operatorname{diag}\left\{2 \xi_{i} \omega_{i}\right\}
$$

then the damped eigenmodes remain uncoupled.

\subsection{Drawbacks of modal truncation}

One can rewrite the system presented in equation (1) in order to obtain the following state-space realization: 


$$
\left\{\begin{aligned}
{\left[\begin{array}{l}
\ddot{q}(t) \\
\dot{q}(t)
\end{array}\right] } & =\overbrace{\left[\begin{array}{cc}
-\mathcal{M}^{-1} \mathcal{C}-\mathcal{M}^{-1} \mathcal{K} \\
I & 0
\end{array}\right]}^{A}\left[\begin{array}{c}
\dot{q}(t) \\
q(t)
\end{array}\right]+\overbrace{\left[\begin{array}{c}
\mathcal{M}^{-1} b \\
0
\end{array}\right]}^{B} u(t) \\
y(t) & =\underbrace{\left[\begin{array}{ll}
c & 0
\end{array}\right]}_{C}\left[\begin{array}{l}
\dot{q}(t) \\
q(t)
\end{array}\right]
\end{aligned}\right.
$$

Assuming that the eigenmodes are uncoupled, the $2 n$ eigenvalues $\lambda_{i}$ of the matrix $A$ are:

$$
\operatorname{eig}(A)=\left\{\lambda_{1,2}=-\xi_{1} \omega_{1} \pm \jmath \omega_{1} \sqrt{1-\xi_{1}^{2}}, \ldots, \lambda_{2 n-1,2 n}=-\xi_{n} \omega_{n} \pm \jmath \omega_{n} \sqrt{1-\xi_{n}^{2}}\right\}
$$

Usually, the aim of modal truncation is to reduce the order of a system by leaving out the eigenmodes corresponding to some criteria enumerated below.

- In the damped case, the eigenmodes to be neglected can be selected according to

(1) the eigenfrequencies (usually the higher ones),

(2) the dynamics (usually the more damped ones),

(3) the $\mathcal{H}_{\infty}$-norm (the smaller ones). Indeed, the transfer function can be written as: $G(s)=\left[\begin{array}{lll}c_{1} \ldots & c_{2 n}\end{array}\right]\left[\begin{array}{ccc}s-\lambda_{1} & & \\ & \ddots & \\ & & s-\lambda_{2 n}\end{array}\right]^{-1}\left[\begin{array}{c}b_{1} \\ \vdots \\ b_{2 n}\end{array}\right]=\sum_{i=1}^{2 n} \frac{c_{i} b_{i}}{s-\lambda_{i}}$.

The $\mathcal{H}_{\infty}$-norm of each term of the sum is given by $\left\|\frac{c_{i} b_{i}}{s-\lambda_{i}}\right\|_{\infty}=\frac{\left|c_{i} b_{i}\right|}{\left|\operatorname{Re} \lambda_{i}\right|}$. So, in order to obtain a small error upper bound in the $\mathcal{H}_{\infty}$-norm, the terms with the smaller $\mathcal{H}_{\infty}$-norm have to be removed.

- In the undamped case (the dissipation matrix is zero: $\mathcal{C}=0$ ), the poles of the system are located on the imaginary axis, therefore the $\mathcal{H}_{\infty}$-norm (or $\mathcal{L}_{\infty}$-norm) and the Hankel singular values cannot be computed. Currently, the only way to perform modal truncation is to leave out the eigenmodes corresponding to some of the eigenfrequencies (usually the higher ones).

As underlined in [21], from a system and control theory point-of-view, in the undamped case, the reduced model may have disadvantages since the analysis is only concerned with the system matrix and not with the input and output matrices. In the following Sections, a new criterion is defined in order to take account of both the system dynamics and the input and output matrices. 


\section{Energy-Based Criterion}

The aim of this Section is to define a new criterion based on the physical energy. Dealing with passive systems $[24,25]$ and focusing on the energy supplied, a natural approach is to use the Hamiltonian framework.

\subsection{Hamiltonian formulation}

Generally, a dissipative Hamiltonian system is defined [20] as

$$
\left\{\begin{array}{l}
\dot{x}(t)=(J(x(t))-R(x(t))) \nabla_{x} \mathcal{H}(x(t))+B(x(t)) u(t) \\
y(t)=B^{t}(x(t)) \nabla_{x} \mathcal{H}(x(t))
\end{array}\right.
$$

with:

- $x=\left[q^{t} p^{t}\right]^{t}$ the state vector.

- $q=\left[q^{1} \ldots q^{n}\right]^{t} \in M$ is the vector of the generalized coordinates, belonging to the configuration manifold $M$ with $\operatorname{dim}(M)=n$.

- $p=\left[p_{1} \ldots p_{n}\right]^{t} \in T_{q}^{*} M$ is the vector of the generalized momentum, belonging to the cotangent space to $M$ at the configuration $q$. Therefore, the vector $x$ belongs to $T^{*} M$ the cotangent bundle(also known as phase space) of $M$ and $\operatorname{dim}(x)=2 n$.

- $\mathcal{H}(x)$ is the Hamiltonian function and represents the total energy of the system,

- $\nabla \mathcal{H}$ represents the column gradient vector.

- $J(x)=-J^{t}(x)$, the symplectic matrix. It is smooth with respect to $x$.

- $R(x)=R^{t}(x) \succeq 0$, the dissipation matrix. It is a smooth function with respect to $x$.

- $B$ is a column vector.

A convenient property of this kind of systems is that the energy balance can be easily obtained:

$$
\begin{aligned}
\frac{\mathrm{d}}{\mathrm{d} t} \mathcal{H}(x(t)) & =\nabla_{x}^{t} \mathcal{H}(x(t)) \dot{x}(t) \\
& =\nabla_{x}^{t} \mathcal{H}(x(t))\left[(J(x(t))-R(x(t))) \nabla_{x} \mathcal{H}(x(t))+B(x(t)) u(t)\right] \\
& =\underbrace{\nabla_{x}^{t} \mathcal{H}(x(t)) J \nabla_{x} \mathcal{H}(x(t))}_{=0}-\nabla_{x}^{t} \mathcal{H}(x(t)) R(x(t)) \nabla_{x} \mathcal{H}(x(t))+\nabla_{x}^{t} \mathcal{H}(x(t)) B(x(t)) u(t) \\
& =y^{t}(t) u(t)-\nabla_{x}^{t} \mathcal{H}(x(t)) R(x(t)) \nabla_{x} \mathcal{H}(x(t))
\end{aligned}
$$

Since $R \succeq 0$, the Hamiltonian systems are passive [20].

In this paper, the focus will only be on linear invariant Hamiltonian systems. In this case, matrices $J, R$ and $B$ are independent of $x$. Moreover, the Hamiltonian function is defined by the following quadratic form: 


$$
\mathcal{H}(x)=\frac{1}{2} x^{t} E x
$$

where $E=E^{t} \succeq 0$ is called the energy matrix.

The system presented in equation (2) has the following state-space realization (called energy variable representation [16]):

$$
\left\{\begin{array}{l}
\dot{x}(t)=(J-R) E x(t)+B u(t) \\
y(t)=B^{t} E x(t)
\end{array}\right.
$$

The Hamiltonian function is defined over the cotangent bundle $T^{*} M$. The generalized velocities $\dot{q}=\left[\begin{array}{lll}\dot{q}^{1} & \ldots & \dot{q}^{n}\end{array}\right]^{t}$ belong to the tangent space to $M$ at the configuration $q: T_{q} M$. In the linear case, the value of the kinetic energy $E_{k}(p)=p^{t} \mathcal{M}^{-1} p$ is equal to the value of the kinetic co-energy $E_{k}^{*}(q)=\dot{q}^{t} \mathcal{M} \dot{q}$ (Legendre transform of $E_{k}$ ). More properties of energy and co-energy can be found in $[16,26]$ for instance.

\subsection{Relation Between the Input Fourier Transform and the Energy Supplied}

Considering the linear Hamiltonian systems, an important relation between the Fourier Transform of the input system with the energy supplied by this input is presented in this Section. To the author's knowledge, this relation has never been presented and used in the related literature. This is the key point on which the new criterion is based.

Consider the undamped harmonic oscillator of the form.

$$
\frac{\mathrm{d}^{2} q}{\mathrm{~d} t^{2}}(t)+\omega_{0}^{2} q(t)=\frac{1}{m} u(t) \quad \text { with } \quad \omega_{0}^{2}=\frac{k}{m} .
$$

Defining $p=m \dot{q}$, the Hamiltonian function is $\mathcal{H}(q, p)=\frac{1}{2 m} p^{2}+\frac{1}{2} k q^{2}=\frac{1}{2} m \dot{q}^{2}+\frac{1}{2} k q^{2}$, this system can be rewritten as equation (2) with $J=\left[\begin{array}{cc}0 & 1 \\ -1 & 0\end{array}\right], R=0$ and $B=\left[\begin{array}{l}0 \\ 1\end{array}\right]$. The energy variable representation is immediately obtained:

$$
\left(\begin{array}{c|c}
A \mid B \\
\hline C \mid D
\end{array}\right)=\left(\begin{array}{cc|c}
{\left[\begin{array}{cc}
0 & \frac{1}{m} \\
-k & 0
\end{array}\right]} & {\left[\begin{array}{l}
0 \\
1
\end{array}\right]} \\
\hline\left[\begin{array}{ll}
0 & \frac{1}{m}
\end{array}\right] & 0
\end{array}\right)
$$


Proposition 3.1 Considering a forcing function (or distribution) $u$ with the support $[0, T]$ (where $T>0$ can be finite or infinite). The energy supplied to an harmonic undamped oscillator given by equation (3) initially at equilibrium with nil mechanical energy is

$$
\mathcal{H}(x(t))=\frac{1}{2 m}\left|U\left(\omega_{0}\right)\right|^{2}=\frac{C B}{2}\left|U\left(\omega_{0}\right)\right|^{2} \quad \forall t \geq T
$$

where $U$ is the Fourier transform of $u$.

Proof:

The oscillator is assumed to be initially in equilibrium with a nil mechanical energy. Therefore, the energy supplied to the system is its mechanical energy at the time $T: \mathcal{H}_{T}$ and remains constant after this time: $\mathcal{H}(t)=\mathcal{H}_{T} \quad \forall t \geq T$.

Defining $y(t)=\dot{q}(t)$, the energy supplied by the input force $u$ is given by its work:

$$
W=\int_{-\infty}^{\infty} y(t) u(t) \mathrm{d} t=\frac{1}{2 \pi} \int_{-\infty}^{\infty} Y(\omega) U^{*}(\omega) \mathrm{d} \omega
$$

where the second equality is obtained by the application of the Parseval theorem and the uppercase variables are the Fourier transforms of the respective lowercase variables.

The derivative property of the Fourier transform leads to $Y(\omega)=\jmath \omega Q(\omega)$ where $Q$ (the Fourier transform of $q$ ) is obtained from the equation (3):

$$
Q(\omega)=\frac{1}{m} \frac{U(\omega)}{\omega_{0}^{2}-\omega^{2}}
$$

The singular points $\left(\omega= \pm \omega_{0}\right)$ lead to a problem in the evaluation of $W$.

To obtain a better definition of the work, the singular points are slightly shifted out of the path of integration through the introduction of an infinitesimally small friction coefficient $\varepsilon>0\left(\varepsilon^{2}\right.$ negligible compared with $\left.\omega_{0}^{2}\right)$. The previous Fourier transforms becomes:

$$
Q_{\varepsilon}(\omega)=\frac{1}{m} \frac{U(\omega)}{\omega_{0}^{2}-(\omega-\jmath \varepsilon)^{2}} \quad \text { and } \quad Y_{\varepsilon}(\omega)=\jmath \omega Q_{\varepsilon}(\omega)=\jmath \omega \frac{1}{m} \frac{U(\omega)}{\omega_{0}^{2}-(\omega-\jmath \varepsilon)^{2}}
$$

Moreover, as $y(t)$ and $u(t)$ are real, their Fourier transforms have an Hermitian symmetry (i.e. $F(-\omega)=F^{*}(\omega)$ ), so:

$$
W_{\varepsilon}=\frac{1}{\pi} \operatorname{Re} \int_{0}^{\infty} Y_{\varepsilon}(\omega) U^{*}(\omega) \mathrm{d} \omega=\frac{1}{m \pi} \operatorname{Re} \int_{0}^{\infty} \frac{\jmath \omega}{\omega_{0}^{2}-(\omega-\jmath \varepsilon)^{2}}|U(\omega)|^{2} \mathrm{~d} \omega
$$

Now, using the so-called Dirac-Plemelj formula (also known as the Sokhotsky-Plemelj 
formula, see [7] for instance):

$$
\lim _{\eta \rightarrow 0^{+}} \frac{1}{x \pm \jmath \eta}=\mathcal{P} \frac{1}{x} \mp \jmath \pi \delta(x)
$$

the following equation is obtained letting $\varepsilon$ to zero:

$$
W=\frac{1}{m} \int_{0}^{\infty} \omega\left(-\delta\left(\omega_{0}+\omega\right) \mathcal{P} \frac{1}{\omega_{0}-\omega}+\delta\left(\omega_{0}-\omega\right) \mathcal{P} \frac{1}{\omega_{0}+\omega}\right)|U(\omega)|^{2} \mathrm{~d} \omega
$$

where the first part of the integral is always zero. Therefore, this leads to:

$$
W=\frac{1}{2 m}\left|U\left(\omega_{0}\right)\right|^{2}
$$

Note that $T$ is the time from which the input is zero. This time can be either finite or infinite.

Several remarks can be made about equation (6):

(1) The work $W$ is always positive or nil, this is consistent with the fact that Hamiltonian systems are passive.

(2) If the control signal is a sinusoidal $u(t)=\sin \left(\omega_{u} t\right)$, its Fourier transform is $U(\omega)=$ $\delta\left(\omega_{u}-\omega\right)$. Therefore, if $\omega_{u}=\omega_{0}$, the supplied energy tends to infinity due to the resonance effect or else the supplied energy is zero.

(3) If the control signal is a unitary impulse $u(t)=\delta(t)$, its Fourier transform is the unitary constant $U(\omega)=1$. The supplied energy is therefore $W=\frac{1}{2 m}$. This is consistent with the shock theory (conservation of the impulse). Indeed, by definition, the impulse conservation is given by: $u_{\text {average }} \Delta t=m \Delta v=1$ (since $u$ is a unitary impulse). Moreover, the velocity before the impact is assumed to be zero so the following relation $v=\frac{1}{m}$ is obtained. Just after the impact, the supplied energy is only the kinetic energy: $E_{k}(v)=\frac{1}{2} m v^{2}=\frac{1}{2 m}=W$.

\subsection{Energy Supplied to Eigenmodes}

Consider the following Hamiltonian system:

$$
\left\{\begin{array}{l}
\dot{x}(t)=J \nabla_{x} \mathcal{H}(x(t))+B u(t) \\
y(t)=B^{t} \nabla_{x} \mathcal{H}(x(t))
\end{array}\right.
$$


with:

$$
x=\left[\begin{array}{l}
q \\
p
\end{array}\right] \quad \text { and } \quad B=\left[\begin{array}{l}
0 \\
b
\end{array}\right]
$$

Let $\Phi$ be the modal matrix transformation defined in Section 2.2 and assume (with no loss of generality) that $\Phi^{t} \Phi=\Phi \Phi^{t}=I_{n}$. Define $\Theta=\operatorname{diag}\{\Phi, \Phi\}$ and consider the following transformation $x=\Theta \tilde{x}$. By underlining that the gradient is a covariant vector $\left(\nabla_{x}=\Theta \nabla_{\Theta^{t} x}=\Theta \nabla_{\tilde{x}}\right)$ and that the modal transformation is a canonical transformation $\left(\Theta^{t} J \Theta=J\right)$, the Hamiltonian system in its modal basis can be written as follows:

$$
\left\{\begin{array}{l}
\dot{\tilde{x}}(t)=J \nabla_{\tilde{x}} \tilde{\mathcal{H}}(\tilde{x}(t))+\tilde{B} u(t) \\
y(t)=\tilde{B}^{t} \nabla_{\tilde{x}} \tilde{\mathcal{H}}(\tilde{x}(t))
\end{array}\right.
$$

with:

$$
\begin{gathered}
\tilde{x}(t)=\left[\begin{array}{c}
\tilde{q} \\
\tilde{p}
\end{array}\right] \quad \text { and } \quad \tilde{B}=\left[\begin{array}{l}
0 \\
\tilde{b}
\end{array}\right]=\Theta^{t} B \\
\tilde{\mathcal{H}}(\tilde{x}(t))=\frac{1}{2} \tilde{x}^{t}(t) \tilde{E} \tilde{x}(t) \quad \text { and } \quad \tilde{E}=\Theta^{t} E \Theta=\left[\begin{array}{cc}
\tilde{\mathcal{K}} & 0 \\
0 & \tilde{\mathcal{M}}^{-1}
\end{array}\right] \\
\tilde{\mathcal{M}}=\operatorname{diag}\left\{\tilde{m}_{1}, \ldots, \tilde{m}_{n}\right\} \quad \text { and } \quad \tilde{\mathcal{K}}=\operatorname{diag}\left\{\tilde{k}_{1}, \ldots, \tilde{k}_{n}\right\}
\end{gathered}
$$

As eigenmodes are orthogonal, the Hamiltonian function $\tilde{\mathcal{H}}$ is the sum of the $n$ Hamiltonian sub-functions $\tilde{\mathcal{H}}_{i}$ of each following Hamiltonian sub-system (written in the energy variable representation):

$$
\left(\begin{array}{c|c}
\tilde{A}_{i} \mid \tilde{B}_{i} \\
\hline \tilde{C}_{i} \mid \tilde{D}_{i}
\end{array}\right)=\left(\begin{array}{cc|c}
{\left[\begin{array}{cc}
0 & \frac{1}{\tilde{m}_{i}} \\
-\tilde{k}_{i} & 0
\end{array}\right]} & {\left[\begin{array}{c}
0 \\
\tilde{b}_{i}
\end{array}\right]} \\
\hline\left[\begin{array}{cc}
0 & \tilde{c}_{i}
\end{array}\right] & 0
\end{array}\right)
$$

where $\tilde{b}_{i}$ is the $i$ th entry of the vector $\tilde{b}$ and $\tilde{c}_{i}=\frac{\tilde{b}_{i}}{\tilde{m}_{i}}$.

Now, using equation (6), $\forall t \geq T$, the $n$ Hamiltonian sub-functions $\tilde{\mathcal{H}}_{i}$ can be written as follows: $\tilde{\mathcal{H}}_{i}\left(\tilde{x}_{i}(t)\right)=\frac{1}{2} C_{i} B_{i}\left|U\left(\omega_{i}\right)\right|^{2}$ (with $\omega_{i}^{2}=\frac{k_{i}}{m_{i}}$ and $\tilde{x}_{i}$ the associated modal statevector). 


$$
\mathcal{H}(x(t))=\sum_{i=1}^{n} \tilde{\mathcal{H}}_{i}\left(\tilde{x}_{i}(t)\right)=\sum_{i=1}^{n} \frac{1}{2} C_{i} B_{i}\left|U\left(\omega_{i}\right)\right|^{2}
$$

\subsection{Example of Modal Energy Computation}

In this section, consider the following system composed of 3 masses chained by 3 springs:

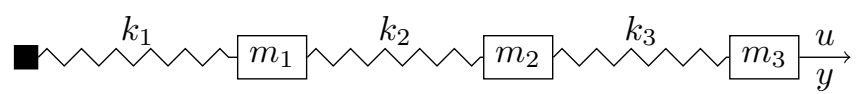

The input is a force $u$ applied to the third mass and the output is the velocity $y$ of the same mass. This system can be described by the following matrices:

$$
\mathcal{M}=\operatorname{diag}\left(m_{1}, m_{2}, m_{3}\right) ; \quad \mathcal{K}=\left[\begin{array}{ccc}
k_{1}+k_{2} & -k_{2} & 0 \\
-k_{2} & k_{2}+k_{3} & -k_{3} \\
0 & -k_{3} & k_{3}
\end{array}\right] ; \quad b=\left[\begin{array}{c}
0 \\
0 \\
1
\end{array}\right] ; \quad \text { and } \quad c=b^{t}
$$

The energy supplied to the 3 modes of the system is computed considering the 2 following cases:

(1) Rectangular input: $u(t)=A \quad \forall 0 \leq t \leq T$ ( $u=0$ elsewhere)

(2) Exponential input: $u(t)=A \exp (-t / t 0) \quad \forall t \geq 0(u=0$ elsewhere $)$

The numerical results were obtained with: $m_{1}=m_{2}=m_{3}=1 \mathrm{~kg}$ and $k_{1}=k_{2}=k_{3}=$ $4 \mathrm{~N} / \mathrm{m}$. The computed eigenfrequencies (in rad/s) are: $\omega_{1}=0.8901, \omega_{2}=2.4940$ and $\omega_{3}=3.6039$.

- In case 1 , the amplitude of the input was set with $A=1$ and the length $T$ of the rectangular input was set at the specific value $T=8 \pi / \omega_{2} \simeq 10.0774 \mathrm{~s}$. Figure 1 shows the outputs of the three modes and the output of the system on the left. On the right, one can observe the energy supplied to the three eigenmodes and to the whole system. The dashed lines refer to the computed values (using equations (6) and (9)) of the 4 Hamiltonian functions of each eigenmode and the system. Note that the energy related to the second eigenmode $\mathcal{H}_{2}$ is zero. This is due to the fact that the length of the rectangular input is a multiple of $2 \pi / \omega_{2}$. Figure 2 shows the modulus of the Bode system transfer function in the upper part and in the lower part one can observe the modulus of the input Fourier transform. The 3 squares on the curve show the values at the 3 eigenfrequencies. For the second eigenfrequency, this value is zero which explains why $\mathcal{H}_{2}$ is equal to zero. 

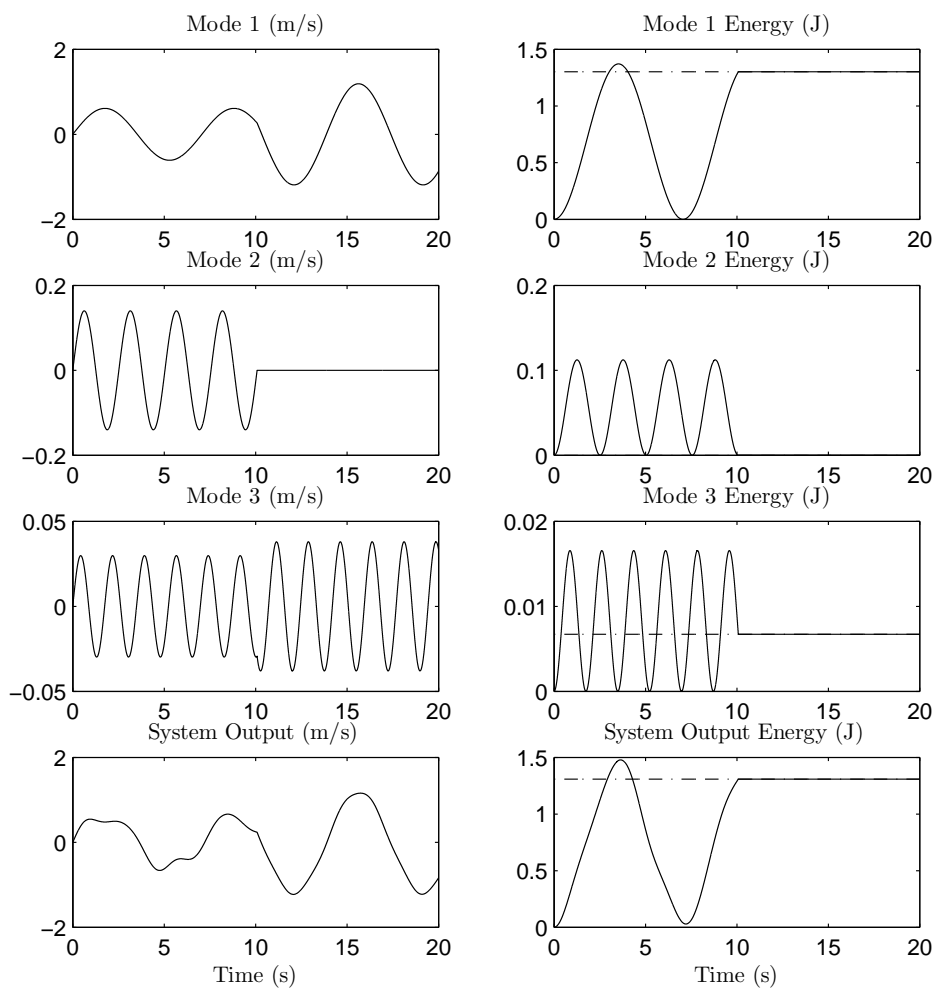

Figure 1. Modal response and modal energy: case 1 (rectangular input).
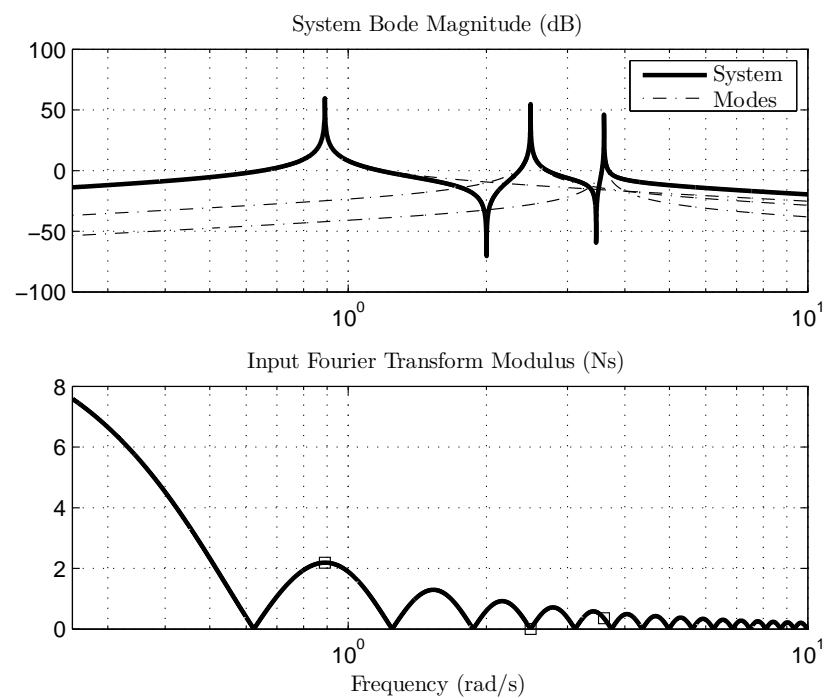

Figure 2. Bode diagram and input Fourier transform: case 1 (rectangular input). 

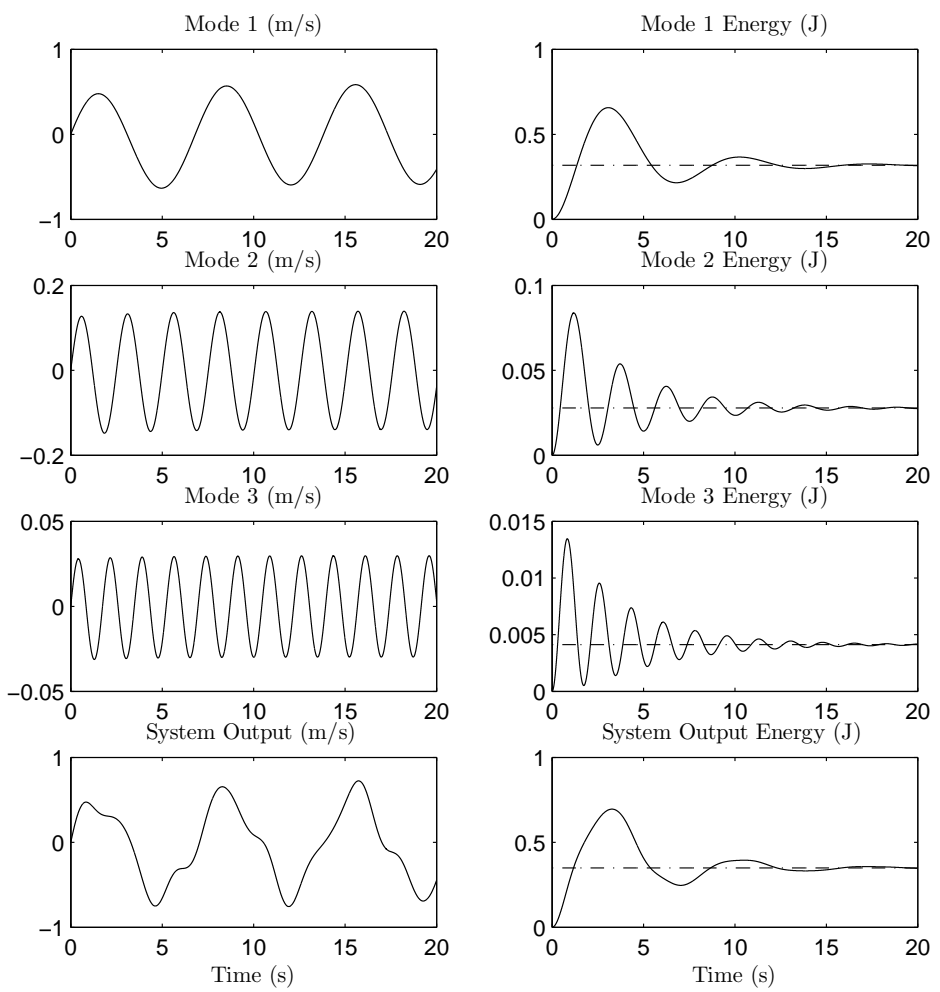

Figure 3. Modal response and modal energy: case 2 (exponential input).

- In case 2 , the amplitude of the input was set with $A=1$ and $t_{0}$ was set at the specific value $t_{0}=4 \mathrm{~s}$. Figure 3 shows the outputs of the three modes and the output of the system on the left. On the right, one can observe the energy supplied to the three eigenmodes and to the whole system. The dashed lines refer to the computed values (using equations (6) and (9)) of the 4 Hamiltonian functions of each eigenmode and the system. 

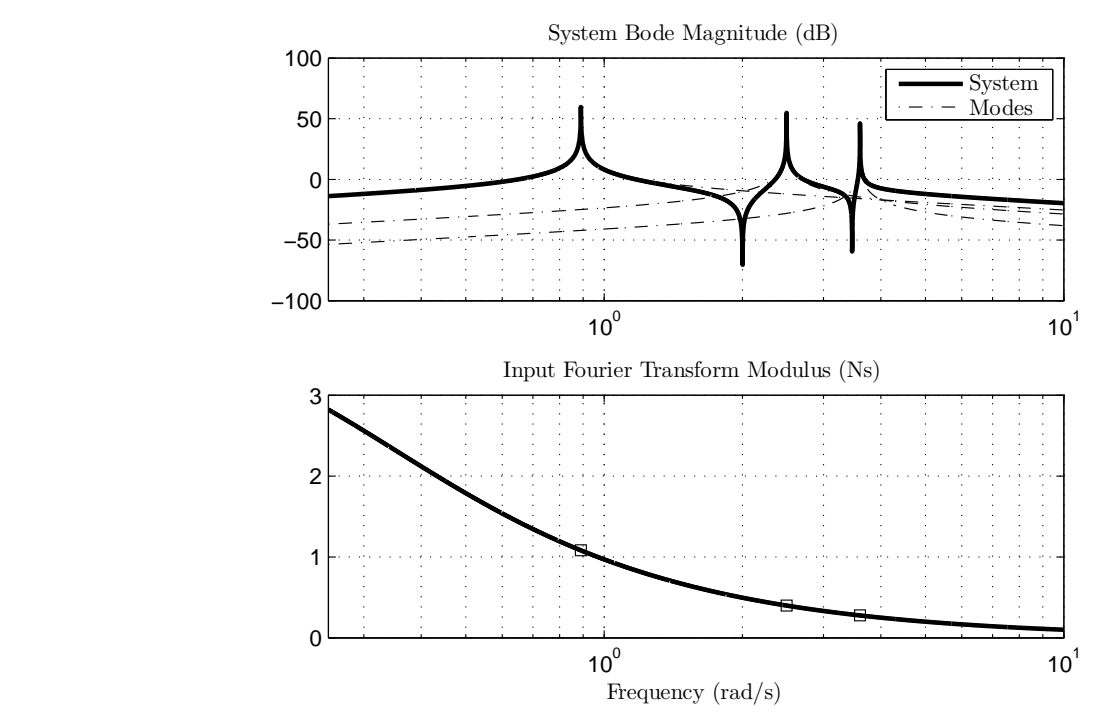

Figure 4. Bode diagram and input Fourier transform: case 2 (exponential input).

Dynamical Systems $\quad$ FinalRevisedMBPaper

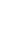




\section{Energy-Based Modal Truncation}

\subsection{Truncation Step}

Physically, it makes sense to keep the most energetic modes, i.e. the modes which have stored most of the energy. This criterion, based on both the eigenfrequency and input/output matrices, is dependent of the type of input signal. If the type of input signal is previously known, its Fourier transform can be directly used to define the criterion and perform a modal truncation in accordingly. In contrast, if there is no knowledge on the type of input signal, the two following cases can be considered for the definition of the criterion:

(1) Dirac (impulse) case: $u(t)=\delta(t) \rightleftharpoons U(\omega)=1$ which leads to

$$
\mathcal{H}(x(t))=\sum_{i=1}^{n} \tilde{\mathcal{H}}_{i}\left(\tilde{x}_{i}(t)\right)=\sum_{i=1}^{n} \frac{1}{2} C_{i} B_{i} \quad \forall t>0
$$

(2) Heaviside (step) case: $u(t)=\Gamma(t) \rightleftharpoons U(\omega)=\mathcal{P} \frac{1}{\jmath \omega}+\frac{1}{2} \delta(\omega)$ which leads to

$$
\mathcal{H}(x(\infty))=\sum_{i=1}^{n} \tilde{\mathcal{H}}_{i}\left(\tilde{x}_{i}(\infty)\right)=\sum_{i=1}^{n} \frac{1}{2} C_{i} B_{i} \frac{1}{\omega_{i}^{2}}
$$

These signals are suggested for two main reasons:

(1) Firstly, these functions are frequently used in system analysis (impulse for the study of the impulse response and step for the study of second order structured system) and reduction. When a balanced truncation is performed on an asymptotic stable system, the reachability gramian $W_{\mathcal{A}}$ considered is solution of the Lyapunov equation $A W_{\mathcal{A}}+W_{\mathcal{A}} A^{t}+B B^{t}=0$ but can also be understood as $W_{\mathcal{A}}=\int_{0}^{\infty} x_{\delta}(\tau) x_{\delta}^{t}(\tau) \mathrm{d} \tau$ where $x_{\delta}$ is the state impulse response. Indeed, $W_{\mathcal{A}}=\int_{0}^{\infty}\left(\int_{0}^{t} \exp (A(t-\tau)) B \delta(\tau) \mathrm{d} \tau\right)\left(\int_{0}^{t} \exp (A(t-\tau)) B \delta(\tau) \mathrm{d} \tau\right)^{t} \mathrm{~d} t=$ $\int_{0}^{\infty} \exp (A t) B B^{t} \exp \left(A^{t} t\right) \mathrm{d} t$

(2) Secondly, when the impulse function is used, the criterion is only based on the input output matrices (independently of the eigenfrequencies since its Fourier transform is a constant). When the primitive of the impulse (namely the step) is used, the Fourier transform can be seen as a penalty function which tends to favorise eigenmodes with small eigenfrequencies. Which can be a trade-off between the proposed approach and the classical approach (remove the higher eigenfrequencies).

Although there is no theoretical link between them, it is worth underlining that there is a strong similarity between the second case (equation (11)) and the truncation criterion based on the $\mathcal{H}_{\infty}$-norm in the damped case. In fact, if the damping matrix is not equal 
to zero, then the modal matrices $A_{i}$ can be written as:

$$
\tilde{A}_{i}=\left[\begin{array}{cc}
0 & \frac{1}{\tilde{m}_{i}} \\
-\tilde{k}_{i} & -2 \xi_{i} \omega_{i}
\end{array}\right]
$$

The $\mathcal{H}_{\infty}$-norm of the $i$ th Hamiltonian sub-system can be approximated by: $\left\|\tilde{G}_{i}\right\|_{\infty} \simeq$ $\frac{1}{2} C_{i} B_{i} \frac{1}{\xi_{i} \omega_{i}}$. Indeed, in the SISO (single input single output) case, the $\mathcal{H}_{\infty}$-norm corresponds to the peak gain of the frequency response. This peak occurs at the pseudoeigenfrequency which is close to the eigenfrequency. Therefore, the following approximation can be made: $\left\|G_{i}\right\|_{\infty} \simeq \sigma_{\max }\left(G_{i}\left(\omega_{i}\right)\right)$. Using the triangle inequality, the following relation, quite similar to the relation (11), is then obtained:

$$
\|G\|_{\infty} \leq \sum_{i=1}^{n}\left\|\tilde{G}_{i}\right\|_{\infty}=\sum_{i=1}^{n} \frac{1}{2} C_{i} B_{i} \frac{1}{\xi_{i} \omega_{i}}
$$

\subsection{Example of Reduction}

Consider a system of the form (7) with

$$
\begin{aligned}
& x=\left[\begin{array}{l}
q \\
p
\end{array}\right] \quad B=\left[\begin{array}{l}
0 \\
b
\end{array}\right] \quad \mathcal{H}(x)=\frac{1}{2} x^{t}\left[\begin{array}{cc}
\mathcal{K} & 0 \\
0 & \mathcal{M}^{-1}
\end{array}\right] x
\end{aligned}
$$

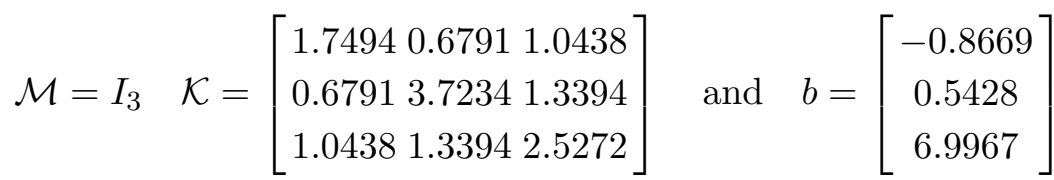

The eigenfrequencies of the system: $\omega_{1}^{2}=1, \omega_{2}^{2}=2$ and $\omega_{3}^{2}=5(\mathrm{in} \mathrm{rad} / \mathrm{s})$ were set arbitrary. The matrices $\mathcal{K}$ and $b$ were generated pseudo-randomly.

The corresponding state-space representation of this system is composed of 6 states (3 generalized positions and 3 generalized velocities). In order to reduce it by removing one eigenmode, two energy-based modal reductions were performed:

(1) The first one was computed using a unitary impulse function (denoted by the superscript $\uparrow$ ). The energy supplied to the 3 eigenmodes is given in the following table:

\begin{tabular}{|ccc|c|c|c|}
\hline Index mode & & $i$ & 1 & 2 & 3 \\
\hline Eigenfrequency & $(\mathrm{rad} / \mathrm{s})$ & $\omega_{i}$ & 1.0000 & 1.4142 & 2.2361 \\
\hline Energy & $(\mathrm{J})$ & $\mathcal{H}_{i}^{\uparrow}$ & 12.4997 & 4.5002 & 8.0000 \\
\hline
\end{tabular}



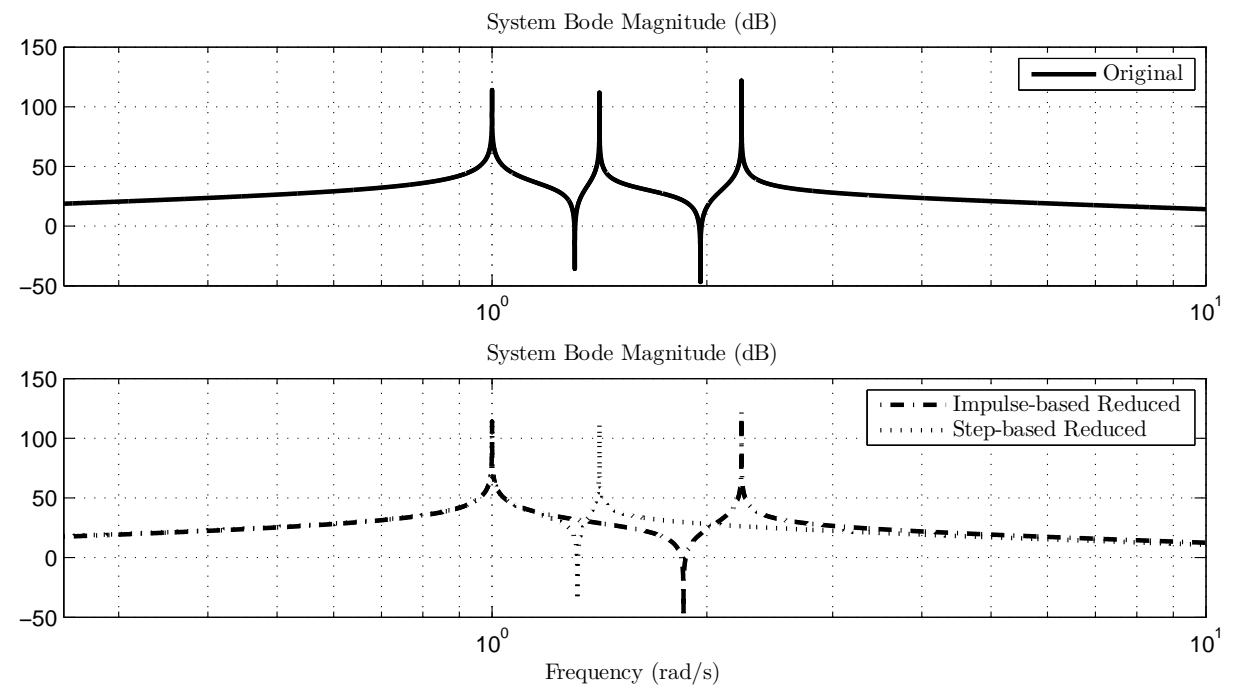

Figure 5. Bode Diagram obtained for impulse-based reduction and step-based reduction.

As $\mathcal{H}_{1}^{\uparrow}>\mathcal{H}_{3}^{\uparrow}>\mathcal{H}_{2}^{\uparrow}$, the eigenmode to be removed is the second one $\left(\mathcal{H}_{2}^{\uparrow}=\right.$ $4.5002 \mathrm{~J})$.

(2) The second one was computed using a Heaviside (step) function with a unitary amplitude (denoted by the superscript $\risingdotseq$ ). The energy supplied to the 3 eigenmodes is given in the following table:

\begin{tabular}{|ccc|c|c|c|}
\hline Index mode & & $i$ & 1 & 2 & 3 \\
\hline Eigenfrequency & $(\mathrm{rad} / \mathrm{s})$ & $\omega_{i}$ & 1.0000 & 1.4142 & 2.2361 \\
\hline Energy & $(\mathrm{J})$ & $\mathcal{H}_{i}^{\triangleright}$ & 12.5003 & 2.2501 & 1.6000 \\
\hline
\end{tabular}

As $\mathcal{H}_{1}^{\gg}>\mathcal{H}_{2}^{\gg}>\mathcal{H}_{3}^{\natural}$, the eigenmode to be removed is the third one $\left(\mathcal{H}_{3}^{\gg}=\right.$ $2.2361 \mathrm{~J})$.

The difference between $\mathcal{H}_{1}^{\uparrow}$ and $\mathcal{H}_{1}^{\longmapsto}$ is only due to numerical approximation.

Figure 5 shows the Bode diagram of the original system and the 2 reduced systems.

In this example, the truncation with respect to a step function gives the same results as the conventional modal truncation, since the Hamiltonian sub-functions are ordered as their corresponding eigenfrequencies. On the other hand, the truncation with respect to an impulse function does not remove the same eigenmodes.

As the $\mathcal{H}_{\infty}$-norm (or $\mathcal{L}_{\infty}$-norm) cannot be computed, the responses to an impulse and a step input are plotted in figure 6 in order to compare the reduced models. 

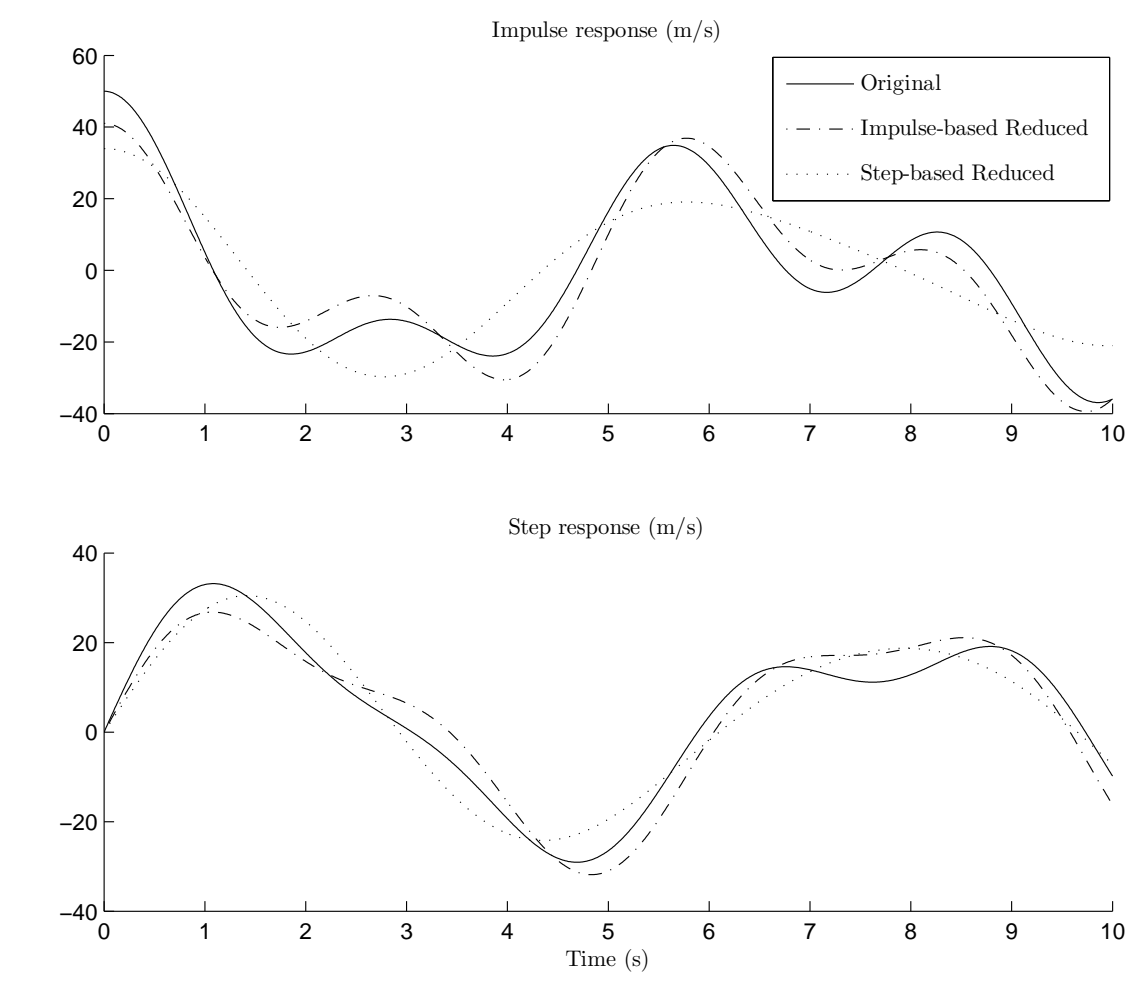

Figure 6. Impulse and Step responses of the original system, impulse-based reduction system and step-based reduction system.

\begin{abstract}
(8)
\end{abstract}




\section{Conclusion}

This paper has discussed the limitations of traditional modal truncation and proposed a new method for the selection of the eigenmodes to be left out. This approach is based on an energetic criterion defined in the same way as the ones for damped system (modal truncation based on the $\mathcal{H}_{\infty}$-norm or balanced truncation based on the Hankel singular values). The new criterions considers the dynamics of the system on the one hand, and, on the other hand the input/output matrices. So, the approach is satisfactory from the point-of-view of a system and control theory. This study focused on conservative systems but the proposed method can be applied on dissipative systems. Further work will propose a trade-off between the energy supplied and the energy dissipated with each eigenmode in order to select the states to be neglected for dissipative systems.

\section{Acknowledgement}

This work was supported by the French national project INOVE/ ANR 2010 BLAN 0308.

\section{References}

[1] A.C. Antoulas. Approximation of Large-scale Dynamical Systems. Cambridge University Press, 2005.

[2] A.C. Antoulas, D.C. Sorensen, and S. Gugercin. A survey of model reduction methods for large-scale systems. Structured Matrices in Operator Theory, Numerical Analysis, Control, Signal and Image Processing, Contemporary Mathematics, AMS publications, 280(6):193-219, 2001.

[3] V.I. Arnold. Mathematical Methods of Classical Mechanics. Springer, 2nd edition, 1989.

[4] T.K. Caughey and M.E.J. O'Kelly. Classical normal modes in damped linear dynamic systems. Transactions of ASME, Journal of Applied Mechanics, 32:583-588, 1965.

[5] Y. Chahlaoui, D. Lemonnier, A. Vandendorpe, and P. van Dooren. Second-order balanced truncation. Linear Algebra and its Applications, 415:373-384, 2006.

[6] R. R. Craig and M. C. C. Bampton. Coupling of substructures for dynamic analyses. American Institute of Aeronautics and Astronautics Journal, 6:1313-1319, 1968.

[7] F.D. Gakhov. Boundary Value Problems. Adiwes International Series in Mathematics, Pergamon Press, 1966.

[8] K. Glover. All optimal Hankel-norm approximation of linear multiariable systems and their $L^{\infty}$-error bounds. International Journal of Control, 39(6):1115-1193, 1984. 
[9] H. Goldstein, C. Poole, and J. Safko. Classical Mechanics. Addison Wesley, 3rd edition, 2000.

[10] R.J. Guyan. Reduction of stiffness and mass matrices. American Institute of Aeronautics and Astronautics Journal, 3(2):380, 1964.

[11] P. Koutsovasilis and M. Beitelschmidt. Comparison of model reduction techniques for large mechanical systems. Multibody System Dynamics, 20:111-128, 2008.

[12] J. Lam, H. Gao, S. Xu, and C. Wang. $H_{\infty}$ and $L_{\infty} / L_{2}$ model reduction for system input with sector nonlinearities. Journal of optimization theory and applications, 125(1):137-155, 2005.

[13] L.D. Landau and E.M. Lifshitz. Mechanics - Third edition. Butterworth Heinemann, 1960.

[14] D. G. Meyer and S. Sirnivasan. Balancing and model reduction for second-order form linear systems. IEEE Transactions on Automatic Control, 41(11):1632-644, 1996.

[15] B.C. Moore. Principal component analysis in linear systems: controllability, observability and model reduction. IEEE Transactions on Automatic Control, 26(1):17-32, 1981.

[16] R.V. Polyuga and A.J. van der Schaft. Structure preserving model reduction of port-hamiltonian systems. In Proc. 18th Int. Symposium on Mathematical Theory of Networks and Systems, Blacksburg, VA, USA, 2008.

[17] J.W.S. Rayleigh. Theory of Sound (2 volumes) 1945th ed. Dover Publications, New York, 3 edition, 1945. 1877.

[18] W.H. Schilders, H.A. van der Vorst, and J. Rommes, editors. Model Order Reduction: Theory, Research Aspects and Applications. Springer, 2008.

[19] T. Stykel. Balanced truncation model reduction of second order systems. In Proceedings of the 5th MATHMOD, February 2006.

[20] A. van der Schaft. Port-Hamiltonian systems: an introductory survey. In Marta Sanz-Sole, Javier Soria, Juan Luis Verona, and Joan Verdura, editors, Proceedings of the International Congress of Mathematicians, Volume III, Invited Lectures, pages 1339-1365, Madrid, Spain, 2006.

[21] A. J. van der Schaft. Model reduction of weakly damped mechanical systems. In Preprints 11th IFAC World Congress, volume 2, pages 277-280, 1990.

[22] A.J. van der Schaft. Time-reversible Hamiltonian systems. Systems 8 Control Letters, 1(5):295-300, 1982.

[23] A.J. van der Schaft. Model reduction of linear conservative mechanical systems. IEEE Transactions on Automatic Control, 35:729-733, 1990.

[24] J.C. Willems. Dissipative dynamical systems I: General theory. Archive for Rational Mechanics and Analysis, 45:321-351, 1972.

[25] J.C. Willems. Dissipative dynamical systems II: Linear systems with quadratic supply rates. Archive for Rational Mechanics and Analysis, 45:352-393, 1972.

[26] H.H. WoodSon and J.R. Melcher. Electromechanical Dynamics-Part I. Wiley, 1968.

[27] P.M. R Wortelboer. Frequency-weighted Balanced Reduction of Closed-loop Mechan- 
ical Servo-systems: Theory and Tools. PhD thesis, University of Delft, 1994.

[28] W-Y. Yan and J. Lam. An approximate approach to $H_{2}$ optimal model reduction.

IEEE Transactions on Automatic Control, 44(7):1341-1358, 1999. IEEE Transetions on Automatic Control, $44(7): 1341-1358,1999$. 